\title{
Starvation ketoacidosis on the acute medical take
}

\author{
Authors: Alexander ] Gall, ${ }^{A}$ Robert Duncan ${ }^{B}$ and Amit Badshah ${ }^{C}$
}

Starvation ketoacidosis (SKA) represents one of three metabolic acidoses caused by the accumulation of ketone bodies within the bloodstream. While easily treated, it is a diagnosis that can be easily missed in patients with an unexplained metabolic acidosis.

In this case report, we discuss two patients presenting with a starvation ketoacidosis and psychiatric illness. This link between SKA and psychiatric disease is especially pertinent as this is a cohort of patients who may not be able to give an accurate history, which can potentially lead to a delay in diagnosis and treatment.

Furthermore, the patient cohort described here have higher rates of alcohol dependence and are therefore at risk of alcoholic ketoacidosis. It is important to recognise that these conditions may coexist and should be managed as such, with thiamine prior to carbohydrate replacement in all at-risk patients.

KEYWORDS: Metabolic acidosis, starvation ketoacidosis, alcoholic ketoacidosis, ketones, mental health

DOI: 10.7861/clinmed.2020-0012

\section{Case presentation}

Here are the cases of two patients presenting on the acute medical take at a district general hospital.

\section{Patient 1}

A 28-year-old man was found on the floor by his mother soaked in his own urine and with a reduced consciousness level. He had intentionally not eaten for 5 days, citing that he wanted to die because people were out to kill him and that he had taken an overdose 10 days prior of 100 modafinil tablets. He had vomited once but denied a cough, breathlessness or chest pain. Past medical history included depression and deliberate self-harm, but

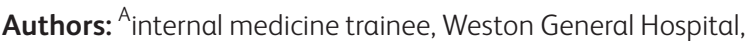
Weston-super-Mare, UK; ${ }^{B}$ foundation year-1 doctor, Weston General Hospital, Weston-super-Mare, UK; ${ }^{C}$ consultant in acute medicine and endocrinology, Weston General Hospital, Westonsuper-Mare, UK the patient was not taking any regular medications and denied any recreational drugs or alcohol.

Initial clinical examination of cardiorespiratory, gastrointestinal and neurological systems were all normal.

Venous blood gas (VBG) sampling revealed $\mathrm{pH}$ of 7.25 , partial pressure of carbon dioxide $\left(\mathrm{pCO}_{2}\right)$ of $5.00 \mathrm{kPa}$, lactate of $1.4 \mathrm{mmol} / \mathrm{L}$, bicarbonate of $16.3 \mathrm{mmol} / \mathrm{L}$, glucose of $3.4 \mathrm{mmol} / \mathrm{L}$ and ketones of $3.3 \mathrm{mmol} / \mathrm{L}$.

\section{Patient 2}

A 43-year-old woman had taken an overdose 4 days prior to admission of 80 co-codamol tablets following an argument with her partner. She was seen by paramedics who did not bring her to hospital as she had been vomiting since and was felt to be low risk. Since then, she had been feeling unwell with ongoing nausea and vomiting, and not eating nor drinking for 3 days. She had a history of alcohol excess but had abstained for 4 days. Past medical history included alcohol excess, anxiety and depression. Regular medication included mirtazapine, co-codamol and propranolol. Examination was once again normal.

Venous blood gas (VBG) sampling revealed $\mathrm{pH}$ of $7.28, \mathrm{pCO}_{2}$ of $3.72 \mathrm{kPa}$, lactate of $<1 \mathrm{mmol} / \mathrm{L}$, bicarbonate of $12.8 \mathrm{mmol} / \mathrm{L}$, glucose of $6.7 \mathrm{mmol} / \mathrm{L}$ and ketones of $4.3 \mathrm{mmol} / \mathrm{L}$.

\section{Diagnosis}

Both patients presented with an euglycaemic ketoacidosis combined with a background of psychiatric disease and current mental health issues. Differentials for an euglycaemic ketoacidosis are:

$>$ starvation ketoacidosis (SKA)

$>$ alcoholic ketoacidosis (AKA)

> euglycaemic diabetic ketoacidosis (DKA; associated with sodium-glucose transport protein- 2 inhibitors, partial treatment with insulin).

Both SKA and AKA are differential diagnoses in these patients, with SKA being the primary differential for patient 1 , and a combination of SKA and AKA being a potential for patient 2 despite reported abstinence in the history. Neither patient had a documented history of diabetes, nor were they taking any antidiabetic medications and euglycaemic DKA was therefore not considered likely in the differentials.

\section{Initial management and prognosis}

The driver for ketone production in patients with SKA is a state of carbohydrate depletion, initial management therefore centres 
around replacing this with intravenous (IV) dextrose. ${ }^{1}$ Patient 1 received $10 \%$ dextrose IV as initial management. Patient 2 was incorrectly identified as having DKA initially and received variable rate insulin plus $10 \%$ dextrose overnight, before recognition of the correct diagnosis during the consultant post-take ward round the following morning.

\section{Case progression and outcome}

The venous blood gas trend of both patients is detailed in Table 1. Patient 1 saw resolution of their acidosis following $3 \mathrm{~L}$ of $10 \%$ dextrose over 10 hours, with potassium replacement. Following medical optimisation, this patient was admitted to the local inpatient psychiatry unit for further work-up and went onto receive a diagnosis of schizophrenia with catatonia requiring electroconvulsive therapy.

Patient 2 was initially managed using the local DKA protocol but saw prompt resolution of their acidosis following recognition of the underlying diagnosis and appropriate management with $10 \%$ dextrose. This patient also received IV thiamine due to concerns regarding overlap of SKA and AKA. Delayed administration of thiamine may account for the slight rise in lactate in the final VBG for patient 2 (Table 1). This patient was reviewed by the local mental health team and discharged to be followed up by their general practitioner.

\section{Discussion}

Starvation ketoacidosis (SKA) represents one of three raised anion gap metabolic acidoses caused by the accumulation of ketone bodies in the blood stream. ${ }^{2}$ The other two causes of a ketoacidosis being alcoholic ketoacidosis and the much more common diabetic ketoacidosis. ${ }^{2}$ Starvation ketoacidosis is important because it is easily missed in patients with an unexplained metabolic acidosis with low/normal blood glucose readings. Furthermore, SKA is simple to manage, but with potential pitfalls in both diagnosis and treatment. Management consists of a combination of IV dextrose, careful monitoring and appropriate replacement of electrolytes. ${ }^{3}$

It is important to screen for SKA in patients with an unexplained metabolic acidosis by checking capillary glucose and ketone measures, both of which are readily available in emergency departments and acute medical wards. Furthermore, one must bear in mind that a lactic acidosis can coexist with a ketoacidosis and therefore consider checking ketones even if lactate is raised. ${ }^{2}$ This is especially relevant if the level of acidosis appears to be out of proportion with the rise in serum lactate.

The patient cohort who are at risk of SKA demonstrates some overlap with those at risk of AKA, including higher rates of mental health problems. ${ }^{4}$ Being aware of this is important in recognising the potential for both SKA and AKA to coexist, as the management differs. ${ }^{2}$ Patients with AKA require administration of IV thiamine prior to commencement of dextrose due to the risk of precipitating a lactic acidosis without thiamine replacement. ${ }^{5}$ This difference is largely academic in the clinical setting and one would not seek to differentiate between diagnoses of SKA and AKA in practise but have a low threshold for administering thiamine replacement prior to commencing dextrose in all at risk patients.

\section{Learning points}

> Remember to check ketones in patients with an unexplained metabolic acidosis.

> Mental health patients are a high-risk group for starvation ketoacidosis.

> There can be overlap between starvation ketoacidosis and alcoholic ketoacidosis.

> Management is simple but requires careful monitoring of fluid status and electrolytes.

$>$ Initial management can cause hypokalaemia due to a physiological surge of insulin.

> Have a low threshold for administration of thiamine replacement in those suspected or at risk of AKA.

Table 1. Resolution of acidosis and ketones levels with administration of intravenous dextrose

\begin{tabular}{|c|c|c|c|c|c|c|c|c|}
\hline \multirow[b]{2}{*}{ Timepoint, hours } & \multicolumn{3}{|c|}{ Patient 1} & \multicolumn{5}{|c|}{ Patient 2} \\
\hline & 0 & 13 & 18 & 0 & 1 & 10 & 13 & 17 \\
\hline $\mathrm{pH}$ & 7.25 & 7.32 & 7.36 & 7.24 & 7.28 & 7.29 & 7.31 & 7.39 \\
\hline $\mathrm{pCO}_{2}, \mathrm{kPa}$ & 5.00 & 4.47 & 4.72 & $\mathrm{n} / \mathrm{a}$ & 3.72 & 4.57 & 4.07 & 5.04 \\
\hline $\mathrm{pO}_{2}, \mathrm{kPa}$ & 9.45 & 13.60 & 13.60 & $n / a$ & 12.89 & 5.07 & 13.45 & 5.96 \\
\hline $\mathrm{Na}^{+}, \mathrm{mmol} / \mathrm{L}$ & 134 & 131 & 133 & $\mathrm{n} / \mathrm{a}$ & 130 & 136 & 133 & 136 \\
\hline $\mathrm{K}^{+}, \mathrm{mmol} / \mathrm{L}$ & 4.42 & 3.08 & 3.51 & $\mathrm{n} / \mathrm{a}$ & 3.66 & 3.86 & 3.50 & 2.93 \\
\hline Lac, mmol/L & 1.4 & 1.3 & $<1$ & $\mathrm{n} / \mathrm{a}$ & $<1$ & $<1$ & $<1$ & 2.1 \\
\hline BE & -10.0 & -7.7 & -4.2 & -11.0 & -12.4 & -9.6 & -9.8 & -2.2 \\
\hline $\mathrm{HCO}_{3,}, \mathrm{mmol} / \mathrm{L}$ & 16.3 & 17.2 & 20.3 & 15.0 & 12.8 & 16.0 & 15.1 & 22.4 \\
\hline $\mathrm{CBG}, \mathrm{mmol} / \mathrm{L}$ & 3.4 & 14.0 & 5.2 & $\mathrm{n} / \mathrm{a}$ & 6.7 & 10.1 & 10.4 & 9.6 \\
\hline Ket, $\mathrm{mmol} / \mathrm{L}$ & 3.3 & 1.6 & 0.8 & $\mathrm{n} / \mathrm{a}$ & 4.3 & 2.4 & $\mathrm{n} / \mathrm{a}$ & 0.9 \\
\hline
\end{tabular}




\section{References}

1 Iwata H, Tsuzuki S, Iwata M, Terasawa T. Ketoacidosis due to a low-carbohydrate diet in an elderly woman with dementia and abnormal eating behavior. Intern Med 2017:56:2671-5.

2 Kraut JA, Madias NE. Metabolic acidosis: Pathophysiology, diagnosis and management. Nat Rev Nephrol 2010;6: 274-85.

3 Yeow C, Wilson F, Walter E, Sultan J. Perioperative diagnosis of euglycaemic ketoacidosis. J Intensive Care Soc 2016;17: 79-81.
4 Awaworyi Churchill S, Farrell L. Alcohol and depression: Evidence from the 2014 health survey for England. Drug Alcohol Depend 2017:180:86-92.

5 Gerrity RS, Pizon AF, King AM, Katz KD, Menke NB. A patient with alcoholic ketoacidosis and profound lactemia. J Emerg Med 2016:51:447-9.

Address for correspondence: Dr Alexander ] Gall, Weston General Hospital, Grange Road, Weston-super-Mare, Somerset BS23 4TQ, UK.

Email: alexander.gall@live.com

\section{We work around the clock because you do}

\section{COVID-19}

We're here to support our members offering the latest advice on the medical-legal issues that the pandemic presents.

\section{themdu.com/coronavirus}

